\title{
MALAT1 knockdown inhibits hypopharyngeal squamous cell carcinoma malignancy by targeting microRNA-194
}

\author{
HONGMING WANG, FEI WANG, WENYU OUYANG, XUEJUN JIANG and WEI LI \\ Department of Otolaryngology, The First Affiliated Hospital of China Medical University, \\ Shenyang, Liaoning 110001, P.R. China
}

Received November 12, 2019; Accepted March 13, 2020

DOI: $10.3892 / \mathrm{ol} .2020 .11551$

\begin{abstract}
Metastasis-associated lung adenocarcinoma transcript 1 (MALAT1) is involved in the oncogenesis and progression of various types of cancer. However, the function of MALAT1 in hypopharyngeal squamous cell carcinoma (HSCC) is not completely understood. In the present study, MALAT1 expression levels were determined using reverse transcription-quantitative PCR, and Cell Counting Kit-8, Transwell and flow cytometry assays were performed to investigate the biological functions of HSCC cells. The results indicated that MALAT1 was upregulated in HSCC. MALAT1 knockdown suppressed HSCC cell proliferation, migration and invasion, and promoted apoptosis compared with the control group. Additionally, microRNA (miR)-194 was identified as a target of MALAT1 and was expressed at low levels in HSCC tissues compared with adjacent non-tumor tissues. A miR-194 agomir inhibited malignant cell behaviors, including cell proliferation, migration and invasion, whereas miR-194 antagomir promoted malignant behaviors compared with the corresponding control groups. In addition, the results suggested that MALAT1 knockdown inhibited the malignant behaviors of HSCC cells by binding miR-194. miR-194 inhibition partially reversed the MALAT1 knockdown-induced inhibitory effects on HSCC cells. Furthermore, MALAT1 knockdown combined with miR194 mimics resulted in the lowest tumor volume among all tested groups in vivo. In conclusion, the results of the present study suggested that MALAT1 knockdown suppressed the malignant behavior of HSCC by targeting miR-194; therefore, MALAT1 may serve as a novel therapeutic target for HSCC.
\end{abstract}

Correspondence to: Dr Wei Li, Department of Otolaryngology, The First Affiliated Hospital of China Medical University, 155 North Nanjing Street, Heping, Shenyang, Liaoning 110001, P.R. China E-mail: wli@cmu.edu.cn

Key words: metastasis associated lung adenocarcinoma transcript 1, hypopharyngeal squamous cell carcinoma, microRNA-194, insulin-like growth factor 1 receptor, yes1-associated transcriptional regulator

\section{Introduction}

Hypopharyngeal squamous cell carcinoma (HSCC) is an aggressive form of head and neck squamous cell carcinoma (HNSCC) that has a poor prognosis (1). Due to a lack of early clinical symptoms, HSCC is often diagnosed at advanced stages, which makes treatment challenging; in addition, the pathogenesis of the disease is not completely understood (2).

An increasing number of studies have focused on the involvement of non-coding RNAs in HSCC with the aim of identifying possible therapeutic strategies $(3,4)$. Metastasis associated lung adenocarcinoma transcript 1 (MALAT1) is a long non-coding (lnc)RNA that has been reported to be involved in neurogenesis (5), metabolic (6) and vascular $(7,8)$ diseases, as well as certain types of cancer (9). MALAT1 affects neuronal differentiation by activating the ERK and mitogen-activated protein kinase (MAPK) signaling pathway in neuro-2a cells (5). MALAT1 has also been reported to be upregulated in a rat model of diabetic gastroparesis, which increases the expression of $\alpha$-smooth muscle actin and smooth muscle myosin heavy chains, and promotes the apoptosis of human gastric smooth muscle cells (6). A previous study also revealed that MALAT1 knockdown inhibited ischemic injury and autophagy by targeting miR-30a in vitro and in vivo (7). Additionally, MALAT1 is upregulated and exhibits oncogenic roles in different types of cancer, including non-small cell lung (10) and pancreatic cancer (11), osteosarcoma (12), hepatocellular (13) and renal cell carcinoma (14), and glioma (15); however, the mechanism underlying MALAT1 activity in HSCC is not completely understood.

microRNAs (miRNAs/miRs) are a type of short non-coding RNAs (18-25 nucleotides in length) that regulate post-transcriptional gene expression by affecting the stability and translation of target mRNAs (15). miR-194 serves roles in metabolic diseases $(16,17)$, dermatosis $(8)$, the inflammatory response (18) and neoplasms $(19,20)$. miR-194 is also involved in multiple aspects of skeletal muscle glucose metabolism by regulating AKT, glycogen synthase kinase 3 and oxidative phosphorylation (16). In a study of psoriasis, transfection with miR-194 mimics suppressed the proliferation and promoted the differentiation of keratinocytes by targeting Grainyhead-like 2 in vitro (8). miR-194 is not only a serum marker of several types of cancer (21), but also functions as a tumor suppressor by regulating malignant behavior of cancer 
cells $(22,23)$. Insulin-like growth factor 1 receptor (IGF1R) and Yes-associated protein 1 (YAP1) have been identified as targets of miR-194 by bioinformatics studies $(24,25)$. IGF1R, a transmembrane receptor tyrosine kinase, participates in the regulation of tumor cell malignancy by activating PI3K and MAPK $(26,27)$. By contrast, YAP1 is an essential regulatory component of the Hippo pathway; YAP1 is involved in various types of malignant behavior, including epithelial-mesenchymal transition, migration, metastasis, and anticancer drug resistance $(28,29)$.

The aim of the present study was to identify the roles of lncRNA MALAT1 and miR-194 in HSCC, as well as the potential underlying mechanism. By studying MALAT1, miR-194, IGF1R and YAP1, the present study aimed to identify novel therapeutic targets for HSCC.

\section{Materials and methods}

Clinical specimens. A total of 25 pairs of human HSCC tissues were obtained from the patients admitted to the Department of Otolaryngology, The First Affiliated Hospital of China Medical University (Shenyang, China) between March 2016 and July 2016. The patients (23 males and 2 females; age, 43-82 years; mean age, 57.8 years) did not receive radiotherapy or chemotherapy prior to surgical resection. HSCC and adjacent non-tumor tissue (ANTT; normal mucosa tissue $\geq 1.5 \mathrm{~cm}$ away from the tumor) specimens were collected from each patient. The present study was approved by the Ethical Committee and Institutional Review Board at The First Affiliated Hospital of China Medical University. Informed consent was obtained from all participants.

Cell culture. FaDu and 293T cells were purchased from The Cell Bank of Type Culture Collection of the Chinese Academy of Sciences. Cells were cultured in DMEM (HyClone; GE Healthcare Life Sciences) containing 10\% FBS (Gibco, Thermo Fisher Scientific, Inc.) at $37^{\circ} \mathrm{C}$ with $5 \% \mathrm{CO}_{2}$.

$R N A$ extraction and reverse transcription-quantitative PCR $(R T-q P C R)$. Total RNA was extracted from the specimens and cells using the TRIzol ${ }^{\circledR}$ reagent (Thermo Fisher Scientific, Inc.) according to the manufacturer's instructions. Total RNA was reverse transcribed into cDNA using the PrimeScript RT reagent kit with gDNA Eraser (Takara Biotechnology Co., Ltd.) or the TaqMan miRNA Reverse Transcription kit (Applied Biosystems; Thermo Fisher Scientific, Inc.). The following conditions were used for reverse transcription: $37^{\circ} \mathrm{C}$ for $15 \mathrm{~min} ; 85^{\circ} \mathrm{C}$ for $5 \mathrm{sec}$; and maintained at $4^{\circ} \mathrm{C}$. Subsequently, MALAT1 and GAPDH expression was quantified by qPCR, using 7500 Fast Real-time PCR (Invitrogen; Thermo Fisher Scientific, Inc.) and SYBR ${ }^{\circledR}$ Premix Ex Taq II (Takara Biotechnology Co., Ltd.). To quantify miR-194 expression levels, qPCR was performed using TaqMan microRNA assays (Applied Biosystems; Thermo Fisher Scientific, Inc.) and TaqMan Universal Master Mix II (Applied Biosystems; Thermo Fisher Scientific, Inc.), as previously described (25). The primer pairs and assays used for qPCR are listed in Tables SI and SII. According to the manufacturer's protocol and referring to previous literature (30), the following thermocycling conditions were used for qPCR: $16^{\circ} \mathrm{C}$ for $30 \mathrm{~min}, 42^{\circ} \mathrm{C}$ for $30 \mathrm{~min} ; 85^{\circ} \mathrm{C}$ for $5 \mathrm{~min}$; and maintained at $4^{\circ} \mathrm{C}$. mRNA and miRNA expression levels were quantified using the $2^{-\Delta \Delta C q}$ method (31), and normalized to the internal reference genes GAPDH and U6, respectively.

Cell transfection. The short hairpin (sh)RNA targeting human MALAT1 was reconstructed in the pGPU6/GFP/Neo vector (sh-MALAT1; Shanghai GenePharma Co., Ltd.). A total of three different sh-MALAT1s were used, each targeting a different sequence. A non-targeting vector was used as the negative control (NC; sh-NC). The sequences of the shRNAs are listed in Table SIII.

Once the cells reached $70 \%$ confluence, they were transfected in 24-well plate using Lipofectamine ${ }^{\circledR} 3000$ reagent (Invitrogen; Thermo Fisher Scientific, Inc.), according to the manufacturer's protocol. The concentration of shRNA was $1 \mathrm{mg} / \mathrm{ml}$. After $48 \mathrm{~h}$, Geneticin (G418; Sigma-Aldrich; Merck KGaA) was chosen to select the stable cell line for $\sim 4$ weeks.

miR-194 agomir (agomir-194) and miR-194 antagomir (antagomir-194; Shanghai GenePharma Co., Ltd.) were used to upregulate and downregulate miR-194 expression, respectively. The sequences were as follows: Agomir-194 forward, 5'-UGU AACAGCAACUCCAUGUGGA-3' and reverse, 5'-CACAUG GAGUUGCUGUUACAUU-3'; and antagomir, 5'-UCCACA UGGAGUUGCUGUUACA-3'; agomir-NC forward, 5'-UUC UCCGAACGUGUCACGUTT-3' and reverse, 5'-ACGUGA CACGUUCGGAGAATT-3'; and antagomir-NC forward, 5'-UUGUACUACACAAAAGUACUG-3'. The reverse strand of agomir-194 had two phosphorothioates at the 5' end, four phosphorothioates and one cholesterol group at the $3^{\prime}$ end, and one full-length nucleotide 2'-methoxy modification. The antagomir sequence had two phosphorothioates at the 5 ' end, four phosphorothioates and one cholesterol group at the $3^{\prime}$ end, and one full-length nucleotide 2'-methoxy modification. Lipofectamine ${ }^{\circledR} 3000$ was used for transfection according to the manufacturer's instructions with the confluency of cells at $50-70 \%$. The final concentration of agomir or antagomir was $30 \mathrm{pmol} / \mathrm{ml}$. After $48 \mathrm{~h}$, cells were collected for subsequent experimentation.

Cell proliferation assay. The Cell Counting Kit- 8 assay (CCK-8; Beyotime Institute of Biotechnology) was used to analyze cell proliferation, according to the manufacturer's instruction. Transfected cells were seeded into 96-well plates $\left(3 \times 10^{3}\right.$ cells/well) and incubated $\left(37^{\circ} \mathrm{C}, 5 \% \mathrm{CO}_{2}\right)$ for $48 \mathrm{~h}$. Subsequently, $10 \mu \mathrm{lCCK}-8$ reagent was added to each well and incubated for another $2 \mathrm{~h}$ at $37^{\circ} \mathrm{C}$. The absorbance of each well was measured at $450 \mathrm{~nm}$ using a microplate reader (Bio-Rad Laboratories, Inc).

Apoptosis analysis. Different groups of cells, including transfected cells and controls were washed with PBS and stained with Annexin V-phycoerythrin/7-aminoactinomycin D (Annexin V-PE/7-AAD Apoptosis Detection kit; Nanjing KeyGen Biotech Co., Ltd.), according to the manufacturer's instructions. Early and late apoptotic cells were analyzed using a FACScan flow cytometer (BD Biosciences). BD CSampler software (version: 1.0.264.21, Becton, Dickinson and Company) was used to analyze the data. 
Cell migration and invasion assays. Transwell plates (diameter, $6.5 \mathrm{~mm}$; pore size, $8 \mu \mathrm{m}$; Costar; Corning, Inc.) were used to assess cell migration and invasion. For the invasion assay, prior to plating the cells into the chambers, the upper chambers were coated with Matrige ${ }^{\circledR}$ and incubated at $37^{\circ} \mathrm{C}$ for $4 \mathrm{~h}$. FaDu cells were seeded $\left(4 \times 10^{5}\right.$ cells $\left./ \mathrm{ml}\right)$ into the upper chamber with $200 \mu \mathrm{l}$ serum-free DMEM. DMEM medium $(600 \mu \mathrm{l})$ containing $10 \% \mathrm{FBS}$ was plated into the lower chambers. Following incubation $\left(37^{\circ} \mathrm{C}, 5 \% \mathrm{CO}_{2}\right)$ for $48 \mathrm{~h}$, the residual cells in the upper chambers were removed using a cotton swab. Cells on the lower surface of the Transwell membrane were stained with $10 \%$ Giemsa (Tiangen Biotech Co., Ltd.) for $2 \mathrm{~h}$ at room temperature. The numbers of stained cells were observed using an Olympus CX43 light microscope (magnification, $\mathrm{x} 400$; Olympus Corporation) in five randomly selected fields of view, and a mean average was calculated.

Luciferase assay. The putative binding sites of miR-194 and MALAT1, IGF1R 3' untranslated region (UTR) or YAP1-3'UTR were obtained using the StarBase (24) and TargetScan (25) databases. The wild-type (WT) or mutant (MUT) binding sequences were cloned into pmirGLO dual-luciferase vectors (Shanghai GenePharma Co., Ltd.). According to the manufacturer's instructions, 293T cells were co-transfected with pmirGLO vector constructed with WT or MUT fragments and agomir-194 or agomir-NC using Lipofectamine ${ }^{\circledR} 3000$ at 50-70\% confluency. After 48-h incubation, the Dual-Luciferase Reporter system (Promega Corporation) was used to analyze luciferase activity, according to the manufacturer's instruction. Firefly luciferase activity was normalized to Renilla luciferase activity.

Western blotting. Total protein was extracted from the cell lysates using RIPA Lysis Buffer with 1\% PMSF (Beyotime Institute of Biotechnology). The protein was determined using a BCA assay (Beyotime Institute of Biotechnology). Equal amounts of protein $(40 \mu \mathrm{g})$ were separated by $10 \%$ SDS-PAGE and transferred to PVDF membranes. Membranes were blocked in a TTBS solution containing $5 \%$ non-fat milk and $0.05 \%$ Tween 20 for $1 \mathrm{~h}$ at room temperature. Then the membranes were incubated at $4^{\circ} \mathrm{C}$ for $12 \mathrm{~h}$ with primary antibodies against IGF1R (cat. no. 20254-1-AP; 1:1,000; ProteinTech Group, Inc.), YAP1 (cat. no. 13584-1-AP; 1:1,000; ProteinTech Group, Inc.) and GAPDH (cat. no. 5174; 1:2,000; Cell Signaling Technology, Inc.). Following primary antibody incubation, the membranes were incubated with a goat anti-rabbit IgG $(\mathrm{H}+\mathrm{L})$, HRP conjugate secondary antibody (cat. no. SA00001-2; 1:1,000; ProteinTech Group, Inc.) at room temperature for $2 \mathrm{~h}$. Protein bands were visualized using an ECL kit (New Cell \& Molecular Biotech Co., Ltd.; http://www. ncmbio.com), and a DNR bio-imaging microchemi system (Neven Yamin, Israel; https://dnr-is.com/products/microchemi). Protein expression levels were quantified using ChemImager 5500 software (version 2.03; ProteinSimple) with GAPDH as the loading control.

Tumor xenograft in nude mice. BALB/C athymic nude mice (age, 4 weeks; weight, 13-15 g) were purchased from the Cancer Institute of Chinese Academy of Medical Science. All animal experiments were approved by the Administrative Panel on Laboratory Animal Care of China Medical
University. The mice were injected with $5 \times 10^{5}$ different groups of FaDu cells into the right armpit. The mice were divided into five groups ( $n=5$ per group): i) Control group receiving the injection of FaDu cells without transfection; ii) sh-NC group; iii) sh-MALAT1 group; iv) agomir-194 group; and v) sh-MALAT1 + agomir-194 group. Tumor volumes were calculated and recorded every four days until 40 days. Tumor volume $\left(\mathrm{mm}^{3}\right)$ was calculated according to the following formula: Volume $=\left(\right.$ length $\mathrm{x}$ width $\left.{ }^{2}\right) / 2$. The humane endpoints were set as follows: i) Tumor diameter $>2.0 \mathrm{~cm}$; ii) ulcerated or necrotic tumor; iii) tumor interfered with normal functions, including eating, ambulating and eliminating; iv) a Body Condition Score (BCS) of 2/5, which indicated that the mouse was underconditioned, with evident segmentation of the vertebral column and readily palpable dorsal pelvic bones; and v) poor overall condition, including displaying signs of pain, lethargy, labored breathing or lack of responsiveness. Mice were euthanized using $\mathrm{CO}_{2}$ with a flow rate of $3 \mathrm{l} / \mathrm{min}$ in a 10-liter cage. Animal death was verified by the absence of heartbeat, response to firm toe pinch and respiratory pattern.

Statistical analysis. Data are presented as the mean \pm SD from at least three independent experiments. Statistical analyses were performed using GraphPad Prism software version 5.0 (GraphPad Software, Inc.). Comparisons between two groups were analyzed using the Student's t-test. Comparisons among multiple groups were analyzed using one-way ANOVA, followed Tukey's post-hoc test. $\mathrm{P}<0.05$ was considered to indicate a statistically significant difference.

\section{Results}

MALAT1 knockdown inhibits HSCC cell proliferation, migration and invasion, and promotes apoptosis. The expression of MALAT1 in ANTT and HSCC tissues was analyzed using RT-qPCR. MALAT1 was significantly upregulated in HSCC tissues compared with ANTT tissues ( $\mathrm{P}<0.05$; Fig. 1A). The efficiency of the three different sh-MALAT1s as assessed, and sh-MALAT1\#1 displayed the highest efficiency among the three shRNAs; therefore, sh-MALAT1\#1 was used for subsequent experiments (Fig. S1A). Following MALAT1 knockdown in the FaDu cell line, HSCC cell proliferation was decreased compared with the control group ( $\mathrm{P}<0.05$; Fig. 1B). Compared with the sh-NC group, cell migration and invasion were significantly decreased in the sh-MALAT1 group $(\mathrm{P}<0.05$; Fig. 1C and D). In addition, the MALAT1 knockdown group displayed increased apoptotic rates compared with those in the sh-NC group $(\mathrm{P}<0.05$; Fig. 1E).

miR-194 targets MALAT1 and functions as a tumor suppressor. The putative binding sites between MALAT1 and miR-194 were predicted using StarBase and TargetScan (Fig. 2A). To verify the interaction between MALAT1 and miR-194, a dual-luciferase reporter assay was performed. Following co-transfection with agomir-194 and pmirGLO-MALAT1-WT, the luciferase activity was decreased compared with that in the agomir-NC and pmirGLO-MALAT1-WT group. Furthermore, the luciferase activity of the agomir-194 and pmirGLO-MALAT1-MUT group was not altered compared with that in the agomir-NC and pmirGLO-MALAT1-MUT 
A

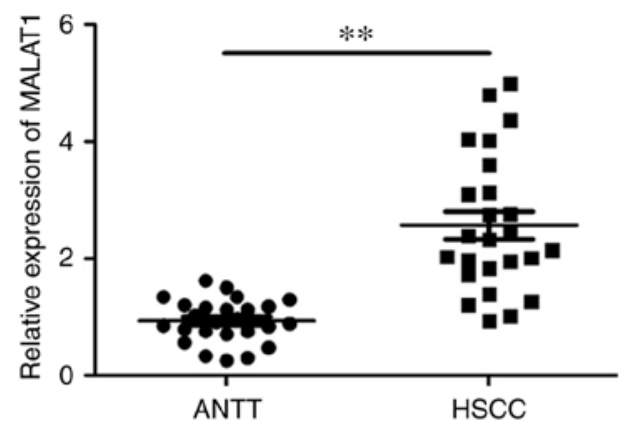

C
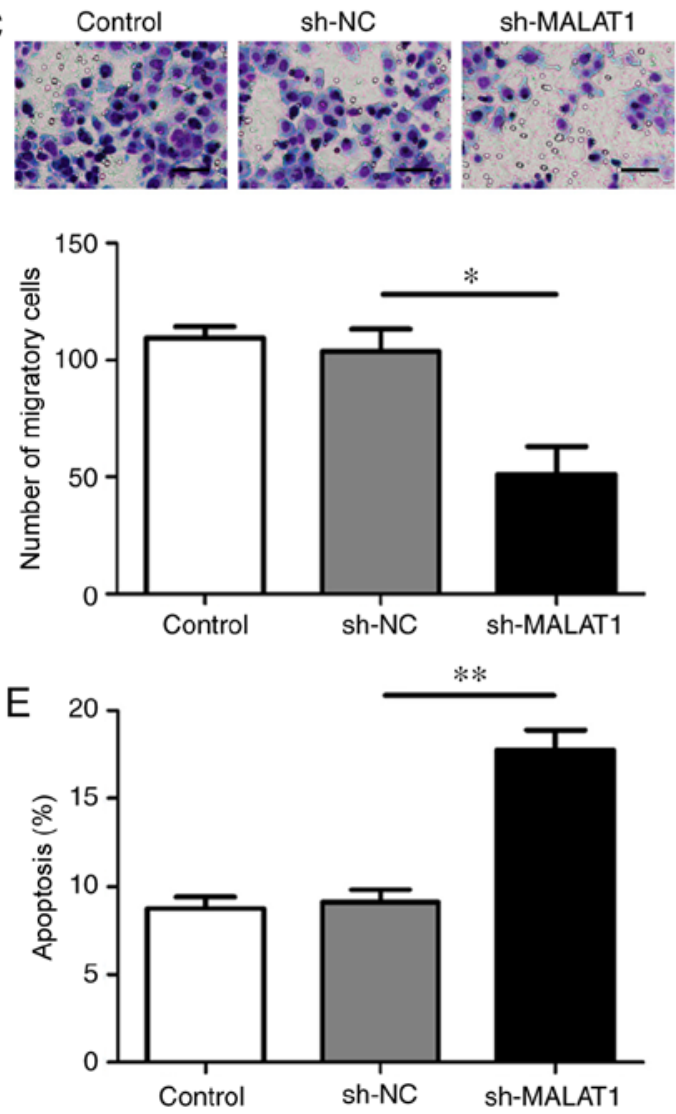

B

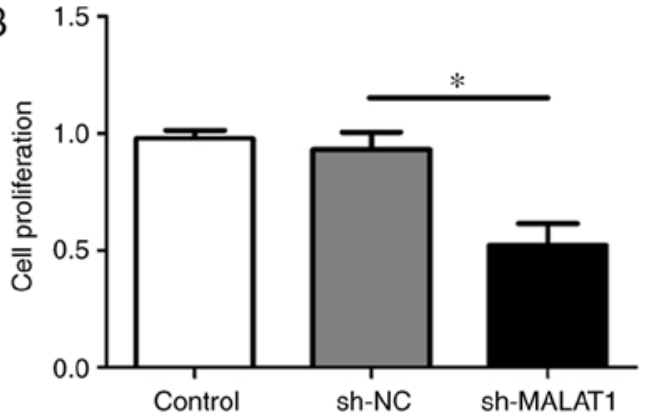

D
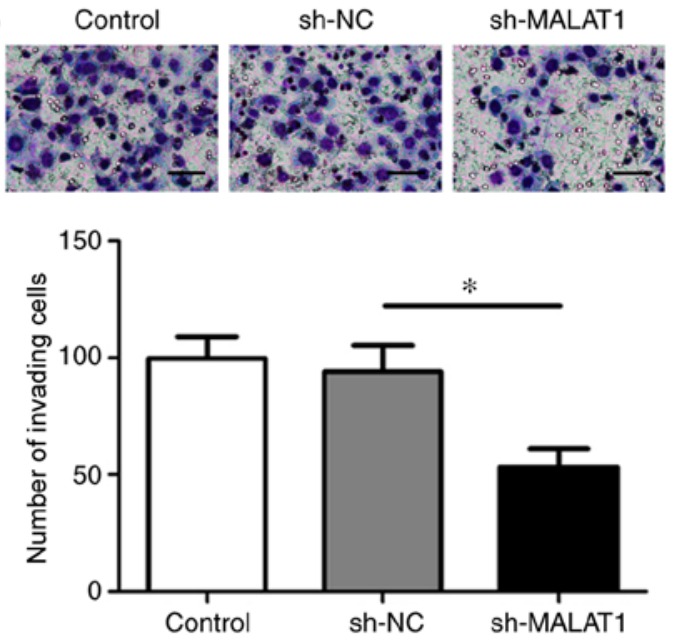

Figure 1. MALAT1 knockdown inhibits HSCC cell proliferation, migration and invasion, and promotes apoptosis. (A) MALAT1 expression in ANTT and HSCC. (B-E) Effects of MALAT1 on FaDu cell (B) proliferation, (C) migration, (D) invasion and (E) apoptosis. Scale bar, $50 \mu \mathrm{m}$. ${ }^{*} \mathrm{P}<0.05$ and ${ }^{* * *} \mathrm{P}<0.01$ as indicated. MALAT1, metastasis-associated lung adenocarcinoma transcript 1; HSCC, hypopharyngeal squamous cell carcinoma; ANTT, adjacent non-tumor tissue; sh, short hairpin RNA; NC, negative control; PE, phycoerythrin; 7AAD, 7-Aminoactinomycin D.

group (Fig. 2B). miR-194 expression was subsequently detected by RT-qPCR. The level of miR-194 expression was downregulated in HSCC compared with ANTT (Fig. 2C).

To evaluate the function of miR-194 in HSCC, agomir-194, antagomir-194 and their respective NCs were transfected into FaDu cells. miR-194 overexpression induced a significant decrease in cell proliferation, migration and invasion, whereas miR-194 inhibition resulted in increased cell proliferation, migration and invasion compared with the corresponding control groups (Fig. 2D and E). In addition, miR-194 overexpression increased the percentage of apoptotic FaDu cells, whereas miR-194 inhibition decreased apoptosis compared with the corresponding control groups (Fig. 2F).

miR-194 mediates the suppressive effects of MALATI knockdown. MALAT1 was identified as a target of
miR-194; therefore, the association between miR-194 and the suppressive effects mediated by MALAT1 knockdown was investigated. sh-MALAT1-transfeced FaDu cells were subsequently co-transfected with agomir-194 or antagomir-194. The result indicated that MALAT1 knockdown decreased cell proliferation, whilst miR-194 overexpression aggravated these effects, compared with the respective controls. As presented in Fig. 3, miR-194 downregulation in the antagomir-194+sh-MALAT1 group partially reversed the aforementioned effects. Similarly, cell migration and invasion were significantly decreased in the sh-MALAT1 + agomir-194 group compared with the sh-NC + agomir-NC group, whereas the inhibitory effects of the sh-MALAT1 group were partially reversed by miR-194 inhibition. Apoptosis was significantly enhanced in the sh-MALAT1 + agomir-194 group compared with 
A malat1-Wt $5^{\prime}$ aUUaguUggcaguggccuguUacg $3^{\prime}$

miR-194 3' AGGUGUACC. IUCAAC. -GACAAUGU $5^{\circ}$

MALAT1-Mut 5 ' AUUAGUGUUCCUGGUCAGUGGCAG $3^{\prime}$
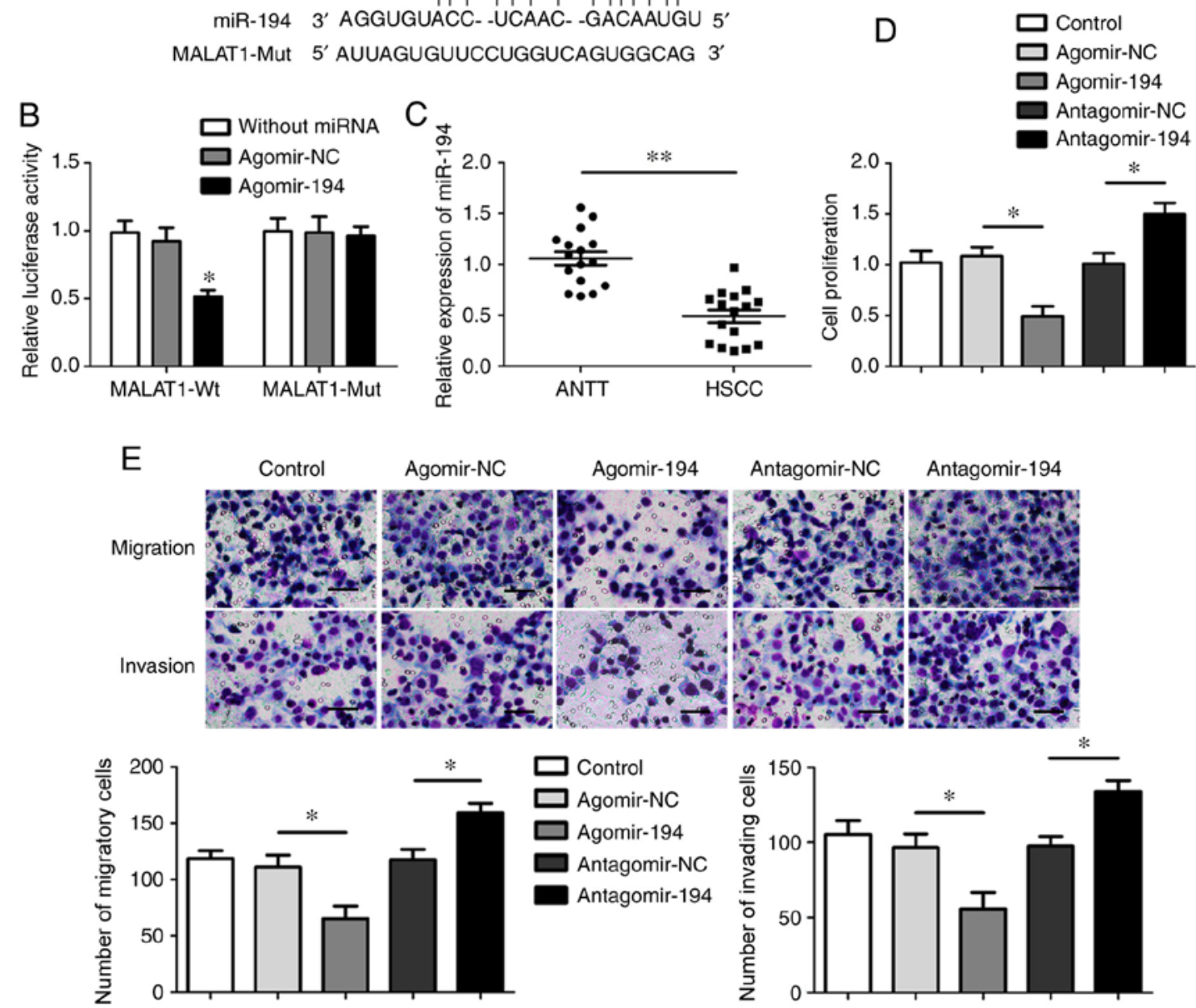

$\mathrm{F}$
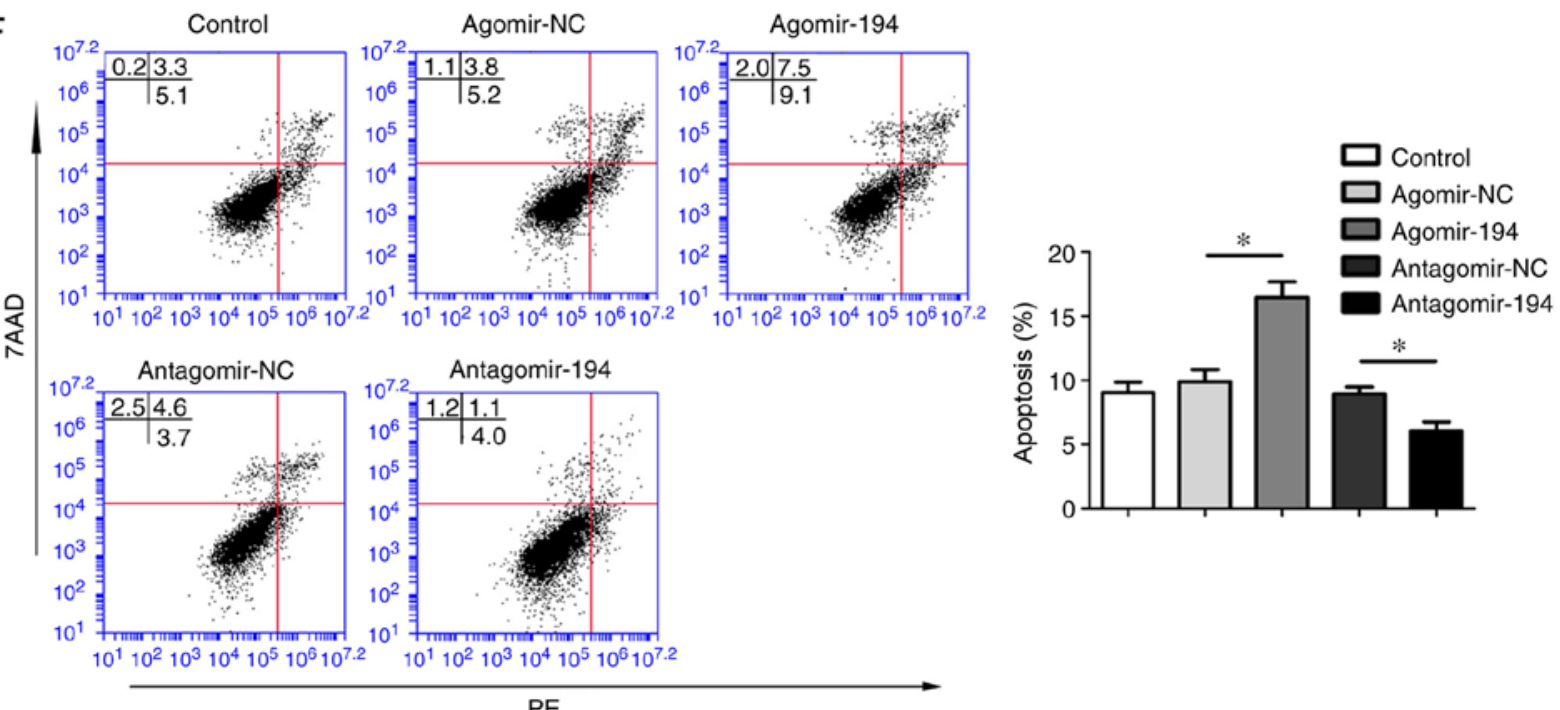

Figure 2. miR-194 targets MALAT1 and functions as a tumor suppressor. (A) The putative binding sites between miR-194 and MALAT1 were predicted by StarBase and TargetScan. (B) Relative luciferase activity in 293T cells was measured using a dual-luciferase reporter assay. (C) miR-194 expression in ANTT and HSCC. ${ }^{* *} \mathrm{P}<0.01$ vs. ANTT. (D-F) Effects of miR-194 on FaDu cell (D) proliferation, (E) migration, invasion and (F) apoptosis. Scale bar, $50 \mu \mathrm{m}$. ${ }^{*} \mathrm{P}<0.05$ and ${ }^{* *} \mathrm{P}<0.01$ vs. MALAT-WT and agomir-NC or as indicated miR, microRNA; MALAT1, metastasis-associated lung adenocarcinoma transcript 1; ANTT, adjacent non-tumor tissue; HSCC, hypopharyngeal squamous cell carcinoma; WT, wild-type; NC, negative control; MUT, mutant; PE, phycoerythrin; 7AAD, 7-Aminoactinomycin D.

the sh-NC + agomir-NC group, and miR-194 inhibition partially reversed the MALAT1 knockdown-mediated effects (Fig. 3A-D).
MALAT1 knockdown decreases the expression of IGFIR and YAP1. The aforementioned results suggested that miR-194 mediated the tumor-suppressive effects of MALAT1 

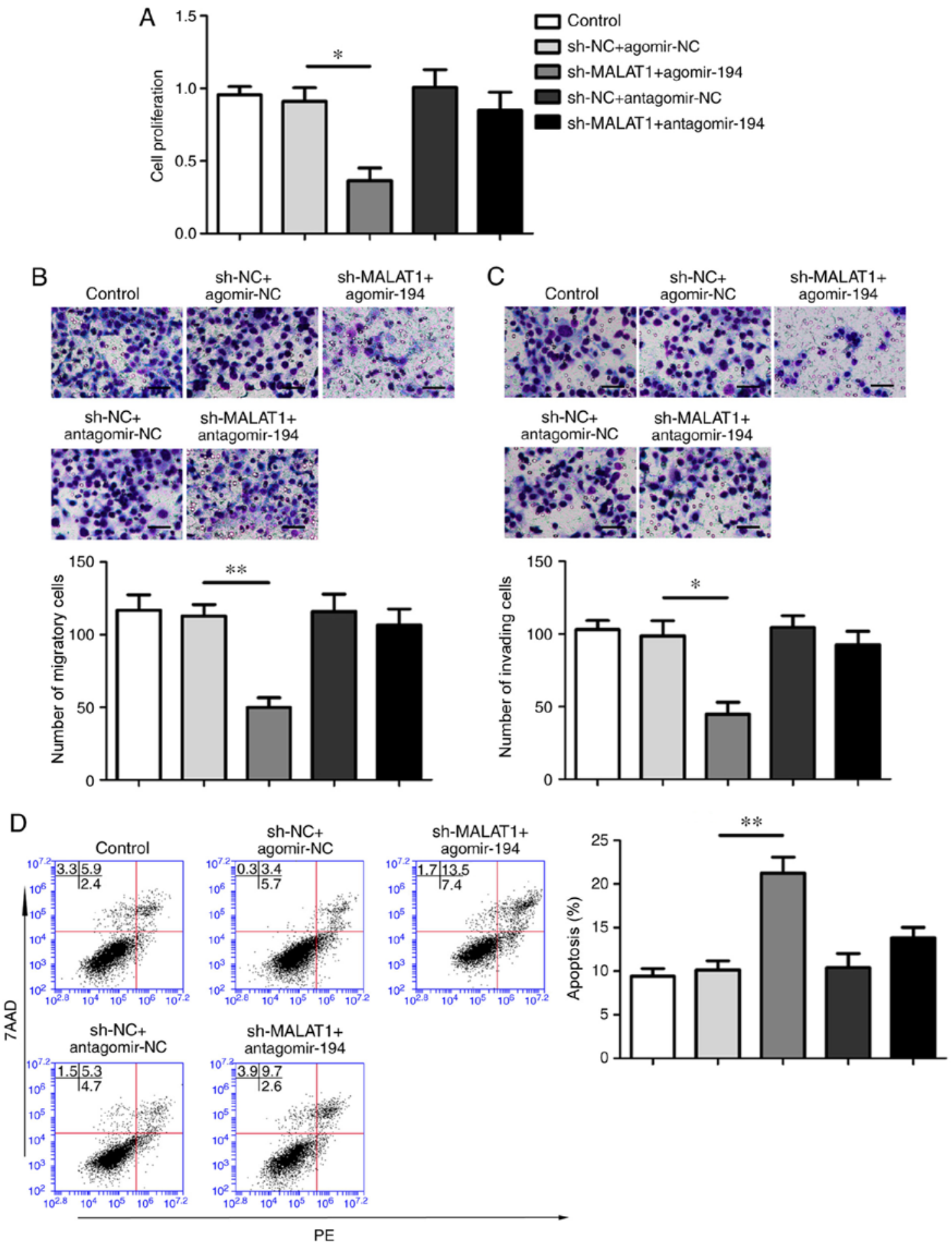

Figure 3. miR-194 mediates MALAT1 knockdown-induced tumor-suppressive effects. (A-E) Effects of MALAT1 knockdown and miR-194 on FaDu cell (A) proliferation, (B) migration, (C) invasion and (D) apoptosis. Scale bar, $50 \mu \mathrm{m}$. ${ }^{*} \mathrm{P}<0.05$ and ${ }^{* *} \mathrm{P}<0.01$. miR, microRNA; MALAT1, metastasis-associated lung adenocarcinoma transcript 1; sh, short hairpin RNA; NC, negative control; PE, phycoerythrin.

knockdown; therefore, it was hypothesized that the downstream targets of miR-194 may be involved in the suppression of malignant behavior.

The binding sites of miR-194 with IGF1R or YAP1 were predicted using the StarBase database and confirmed using a dual-luciferase reporter assay (Fig. 4A and B). The luciferase activity of the agomir-194+IGF1R-3'UTR-WT group decreased compared with the agomir-194+IGF1R-3'UTR-MUT and the agomir-NC+IGF1R-3'UTR-WT groups (Fig. 4A). The binding effects and sites between miR-194 and YAP1-3'UTR were also verified; the results revealed that luciferase activity decreased in the agomir-194+YAP1-3'UTR-WT group compared with the agomir-194+YAP1-3'UTR-MUT and the agomir-NC + YAP1-3'UTR-WT groups (Fig. 4B).

The protein expression levels of IGF1R and YAP1 were assessed using western blotting. miR-194 overexpression significantly decreased the expression levels of IGF1R and YAP1 compared with the agomir-NC group, whereas miR-194 
A IGF1R-Wt 5' CAGCAUUGGAGC---.-CUGUUACA $3^{\prime}$ miR-194 3' AGGUGUACCUCAACGACAAUGU $5^{\prime}$ IGF1R-Mut $5^{\prime}$ CAGCAUGUUCUC.-.-AGUGGCAC 3'

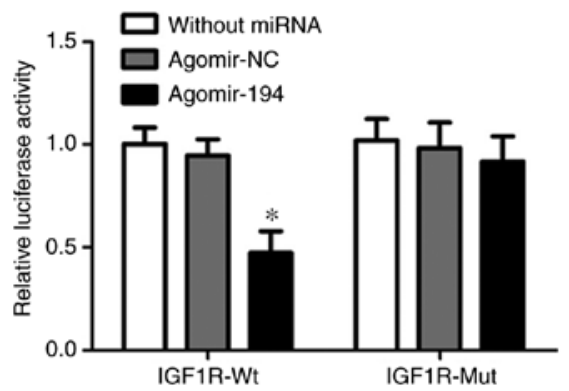

C
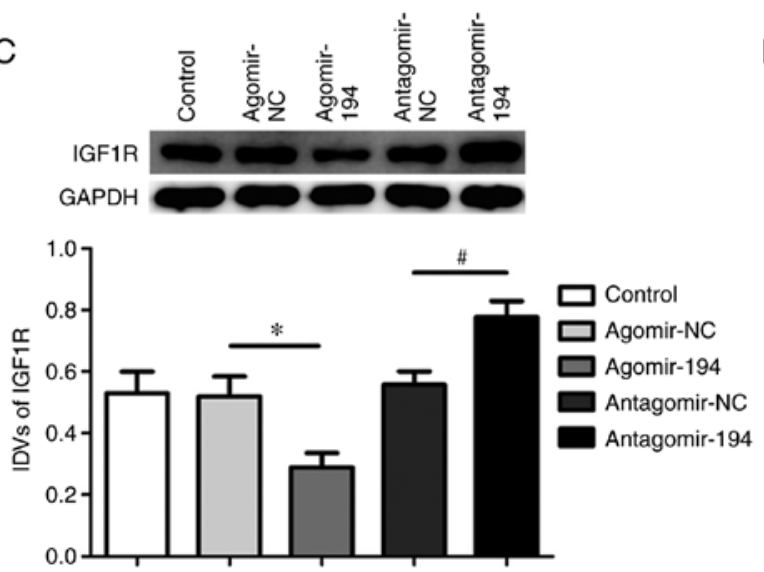

E
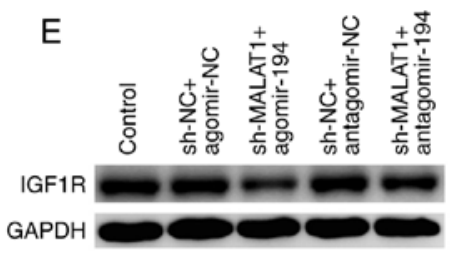

GAPD

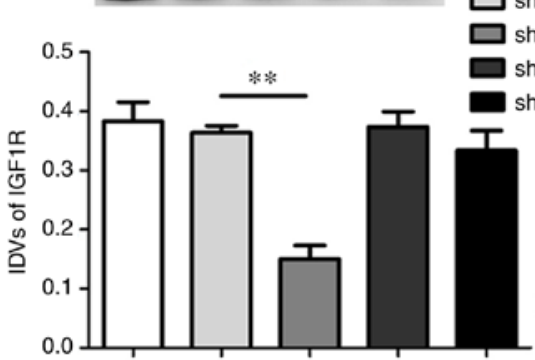

G

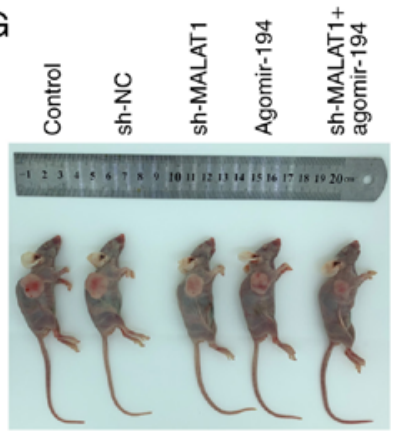

B YAP1-Wt 5' GGUACUUACAUAAAUUGUUACA $3^{\prime}$ miR-194 3' AGgUGUACCUCAACGACAAUGU 5' YAP1-Mut $5^{\prime}$ GGUACUUACAUAAAUGUGGCAC $3^{\prime}$
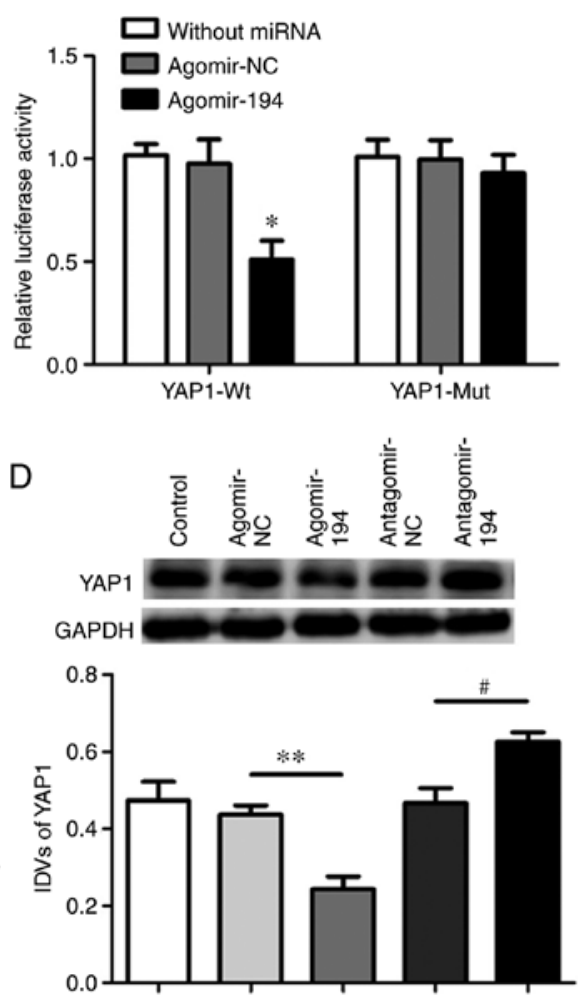

$\mathrm{F}$
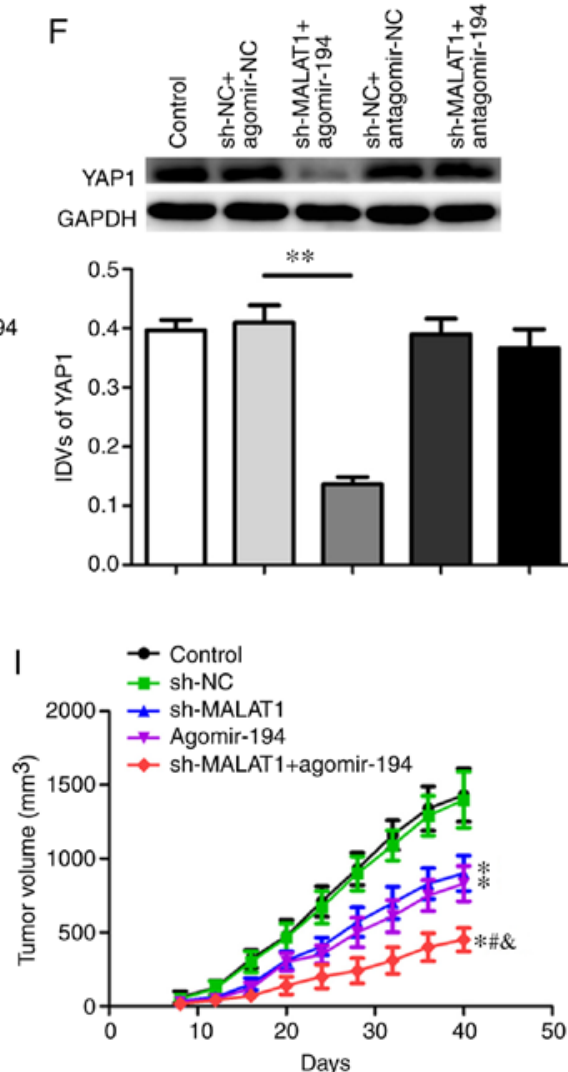

Figure 4. MALAT1 knockdown decreases the expression of IGF1R and YAP1 by upregulating miR-194. (A and B) Relative luciferase activity of 293T cells co-transfected with agomir-NC or agomir-194 and (A) IGF1R-WT or IGF1R-MUT or (B) YAP1-WT or YAP1-MUT were measured using a dual-luciferase reporter assay. "P $<0.05$ vs. IGF1R-WT and agomir-NC or YAP1-WT and agomir-NC. (C and D) Effects of miR-194 on the protein expression of (C) IGF1R and (D) YAP1. ${ }^{\mathrm{P}}<0.05$ and ${ }^{* * *} \mathrm{P}<0.01$; ${ }^{*} \mathrm{P}<0.05$. (E and F) Effects of MALAT1 knockdown combined with miR-194 on the expression of (E) IGF1R and (F) YAP1 ${ }^{* *} \mathrm{P}<0.01$ as indicated. $(\mathrm{G}$ and $\mathrm{H})$ Representative images of the $(\mathrm{G})$ mice and $(\mathrm{H})$ excised tumors. (I) The volume of tumor xenografts. ${ }^{*} \mathrm{P}<0.05$ vs. sh-NC; ${ }^{*} \mathrm{P}<0.05$ vs. sh-MALAT1; ${ }^{\circledR} \mathrm{P}<0.05$ vs. agomir-194. MALAT1, metastasis-associated lung adenocarcinoma transcript 1; IGF1R, insulin-like growth factor 1 receptor; YAP1, yes-associated protein 1; miR, microRNA; WT, wild-type; MUT, mutant; NC, negative control; sh, short hairpin RNA; IDV, integrated density value. 
inhibition significantly increased the expression levels of IGF1R and YAP1 compared with the antagomir-NC group (Fig. 4C and D). The sh-MALAT1 + agomir-194 group exhibited reduced expression levels of IGF1R and YAP1 than controls. IGF1R and YAP1 expression in the sh-MALAT1 + agomir-194 group were lower compared with the controls (Fig. 4E and F).

MALAT1 knockdown combined with miR-194 overexpression inhibits tumor growth. In the xenograft experiments, the tumor volume in the right armpit of each nude mice was calculated every four days. During the study, three mice reached the humane endpoints; two displayed a BCS of $2 / 5$ and one displayed an ulcerated tumor. The largest tumor was observed in the control group, with following dimensions: $\left(1.65 \times 1.45^{2}\right) / 2=1,734 \mathrm{~mm}^{3}$. No mice displayed multiple tumors. The tumor volume of the sh-NC group was not significantly different compared with that of the control group. The tumor volumes of the sh-MALAT1 and agomir-194 groups were decreased compared with the sh-NC group. The lowest tumor volume was observed in the sh-MALAT1 + agomir-194 group (Fig. 4G-I). The tumor volume in the sh-MALAT1 + agomir-194 group significantly decreased compared with the sh-NC, sh-MALAT1 and agomir-194 groups.

\section{Discussion}

In the present study, MALAT1 was upregulated in HSCC tissues compared with ANTT, and MALAT1 knockdown inhibited HSCC cell proliferation, migration and invasion, and promoted apoptosis. miR-194 was identified as a target of MALAT1 and was expressed at low levels in HSCC compared with ANTT specimens. By contrast, downregulation of miR-194 promoted various cellular malignant behaviors, whereas miR-194 overexpression inhibited malignant behaviors. Furthermore, the results suggested that MALAT1 inhibited the malignant behaviors of HSCC cells by binding miR-194, and miR-194 inhibition reversed the MALAT1 knockdown-induced inhibitory effects. Although a wound healing assay was not performed in the present study to verify the migratory ability of HSCC cells, the results of the Transwell assays suggested that MALAT1 and miR-194 altered the biological behaviors of HSCC cells. Binding sites between IGF1R or YAP1 3'-UTRs and miR-194 were also identified, and the expression levels of IGF1R and YAP1 were downregulated following miR-194 overexpression or MALAT1 knockdown compared with the respective control groups. In addition, MALAT1 knockdown combined with miR-194 overexpression resulted in the smallest tumor volume in vivo among all tested groups.

Previously, IncRNAs were considered to serve no functions; however, increasing evidence has demonstrated that lncRNAs are involved in the tumorigenesis and progression of various neoplasms $(32,33)$. The results of the present study indicated that MALAT1 was highly expressed in HSCC specimens. In addition, MALAT1 knockdown in FaDu cells inhibited malignancy in vitro, indicating that MALAT1 may display an oncogenic role during the development of HSCC. Consistent with the present study, MALAT1 is upregulated and functions as oncogene in various types of neoplasm, including non-small cell lung (34), gastric (35) and ovarian cancer (36), as well as oral squamous cell carcinoma (37) and multiple myeloma (38). Different mechanisms underlying MALAT1 activity in the aforementioned types of cancer have been demonstrated. MALAT1 is regulated by transcriptional factor forkhead box $\mathrm{O} 1$ and promotes the malignancy of osteosarcoma cells by inhibiting miR-26a-5p (12). In addition, MALAT1 facilitates vasculogenic mimicry and angiogenesis in gastric cancer via the vascular E-cadherin/ $\beta$-catenin complex, ERK/matrix metallopeptidase and focal adhesion kinase/paxillin signaling pathways (34). MALAT1 knockdown affects the distant metastasis of tongue squamous cell carcinoma by upregulating specific small proline-rich proteins (39). In addition, MALAT1 knockdown inhibits ELAV-like RNA binding protein 1 (HuR)-TIA1 cytotoxic granule associated RNA binding protein (TIA-1)-mediated autophagic activation by interacting with HuR and TIA-1 in pancreatic cancer (40). MALAT1 has been identified as a biomarker for the progression and prognosis of esophageal cancer (41), breast cancer (42) and colorectal cancer (43); therefore, MALAT1 may serve as a potential target for cancer or adjunctive therapy. However, MALAT1 overexpression enhances temozolomide resistance of glioma by upregulating zinc finger E-box binding homeobox (44).

Recently, miR-194 was reported to be a tumor suppressor gene in the majority of cancer types, and low-level miR-194 expression has been associated with high degrees of malignancy (45). miR-194 overexpression inhibits colorectal cancer cell proliferation and invasion by regulating the downstream target transforming growth factor (46). Additionally, miR-194 overexpression negatively regulates AKT serine/threonine kinase 2, which promotes gastric cancer cell proliferation and invasion (47). Following miR-194 suppression, the proliferation of breast cancer cell lines was significantly increased (48). The results of the present study were consistent with the aforementioned studies, suggesting that miR-194 was downregulated in HSCC and served as a tumor suppressor.

Reciprocal inhibition mechanisms between lncRNAs and miRNAs have been demonstrated in previous studies, indicating that lncRNAs sponge miRNAs to prevent downstream binding to mRNAs (49). Therefore, IncRNAs participate in the physiology and pathology of cells by regulating miRNAs levels (50). In the present study, miR-194 was predicted as a target of MALAT1, and a dual-luciferase reporter assay was performed to verify the interaction between miR-194 and MALAT1. The results revealed that altered expression levels of miR-194 involved MALAT1 knockdown-mediated malignant behaviors. miR-194 upregulation inhibited MALAT1 knockdown-mediated malignant behaviors. MALAT1 knockdown combined with miR-194 overexpression significantly suppressed HSCC cell proliferation and promoted HSCC cell apoptosis compared with MALAT1 knockdown alone. Consistent with the results of the present study, it has been reported that MALAT1 sponges miR-503 to modulate epithelial-mesenchymal transition during silica-induced pulmonary fibrosis (51). In ovarian cancer, MALAT1 serves an oncogenic role by sponging miR-200c to promote malignant behavior (36).

miRNAs target genes by binding to their 3'UTRs (52). In the present study, IGF1R and YAP1 were predicted to be the downstream targets of miR-194 using bioinformatics tools. The binding effects and sites were investigated using 
a dual-luciferase reporter assay. The results revealed that miR-194 overexpression reduced the expression of IGF1R and YAP1, whereas miR-194 inhibition increased IGF1R and YAP1 expression compared with the corresponding control groups. IGF1R exerts oncogenic roles in $\operatorname{HNSCC}(53,54)$, and IGF1R knockdown suppresses prostate cancer cell proliferation, migration and invasion (55). IGF1R is also associated with a poor prognosis in patients with gastric and breast cancer $(56,57)$. Additionally, it has been revealed that IGF1R is overexpressed in laryngeal squamous cell carcinoma tissues (58), and IGF1R knockdown enhances radiation sensitivity in human esophageal squamous cell carcinoma (59). YAP1 activation is also associated with a poor prognosis in patients with HNSCC (60), and in head and neck cancer, YAP1 upregulation is associated with cetuximab resistance (61).

In conclusion, the present study investigated the role and effects of MALAT1 in HSCC. MALAT1 knockdown suppressed malignant behaviors in vitro by targeting miR-194. Therefore, MALAT1 and miR-194 may serve as novel therapeutic targets for HSCC.

\section{Acknowledgements}

Not applicable.

\section{Funding}

The present study was funded by The Natural Science Foundation of Liaoning (grant nos. 20180540097 and 201602881)

\section{Availability of data and materials}

All data generated or analyzed during the present study are included in this published article.

\section{Authors' contributions}

HW, XJ and WL designed the present study and performed the experiments. FW and WO collected the data, while HW, WO and FW analyzed the data. HW and XJ drafted the initial manuscript. All authors read and approved the final manuscript.

\section{Ethics approval and consent to participate}

The present study was approved by the Ethics Committee of The Frist Affiliated Hospital of China Medical University (Shenyang, China; approval no. 2016-129) and the Animal Ethics Committee of China Medical University Animal Center (Shenyang, China; approval no. 2017111). Written informed consent was provided by all patients and/or guardians prior to the study start.

\section{Patient consent for publication}

Not applicable.

\section{Competing interests}

The authors declare that they have no competing interests.

\section{References}

1. Newman JR, Connolly TM, Illing EA, Kilgore ML, Locher JL and Carroll WR: Survival trends in hypopharyngeal cancer: A population-based review. Laryngoscope 125: 624-629, 2015.

2. Chan JY and Wei WI: Current management strategy of hypopharyngeal carcinoma. Auris Nasus Larynx 40: 2-6, 2013.

3. Qian Y, Liu D, Cao S, Tao Y, Wei D, Li W, Li G, Pan X and Lei D: Upregulation of the long noncoding RNA UCA1 affects the proliferation, invasion, and survival of hypopharyngeal carcinoma. Mol Cancer 16: 68, 2017.

4. $\mathrm{Xu} \mathrm{S}$, Hui L, Yang $\mathrm{N}$, Wang $\mathrm{Y}$, Zhao $\mathrm{N}$ and Jiang $\mathrm{XJ}$ : Upregulation of microRNA-194-5p inhibits hypopharyngeal carcinoma cell proliferation, migration and invasion by targeting SMURF1 via the mTOR signaling pathway. Int J Oncol 54: 1245-1255, 2019.

5. Chen L, Feng PM, Zhu X, He SX, Duan JL and Zhou D: Long non-coding RNA Malat1 promotes neurite outgrowth through activation of ERK/MAPK signalling pathway in N2a cells. J Cell Mol Med 20: 2102-2110, 2016.

6. Gong Y, Zhu Y, Zhu B, Si X, Heng D, Tang Y, Sun X and Lin L: LncRNA MALAT1 is up-regulated in diabetic gastroparesis and involved in high-glucose-induced cellular processes in human gastric smooth muscle cells. Biochem Biophys Res Commun 496: 401-406, 2018.

7. Guo D, Ma J, Yan L,Li T, Li Z, Han X and Shui S: Down-regulation of Lncrna MALAT1 attenuates neuronal cell death through suppressing Beclin1-dependent autophagy by regulating Mir-30a in cerebral ischemic stroke. Cell Physiol Biochem 43: 182-194, 2017.

8. Yu X, An J, Hua Y, Li Z, Yan N, Fan W and Su C: MicroRNA-194 regulates keratinocyte proliferation and differentiation by targeting Grainyhead-like 2 in psoriasis. Pathol Res Pract 213: 89-97, 2017.

9. Liu J, Peng WX, Mo YY and Luo D: MALAT1-mediated tumorigenesis. Front Biosci (Landmark Ed) 22: 66-80, 2017.

10. Ma J, Wu K, Liu K and Miao R: Effects of MALAT1 on proliferation and apo- ptosis of human non-small cell lung cancer A549 cells in vitro and tumor xenograft growth in vivo by modulating autophagy. Cancer Biomark 22: 63-72, 2018.

11. Wang CJ, Shi SB, Tian J, Xu J and Niu ZX: lncRNA MALAT1, HOTTIP and PVT1 as predictors for predicting the efficacy of GEM based chemotherapy in first-line treatment of pancreatic cancer patients. Oncotarget 8: 95108-95115, 2017.

12. Wang J and Sun G: FOXO1-MALAT1-miR-26a-5p feedback loop mediates proliferation and migration in osteosarcoma cells. Oncol Res 25: 1517-1527, 2017.

13. Chen L, Yao H, Wang K and Liu X: Long non-coding RNA MALAT1 regulates ZEB1 expression by sponging miR-143-3p and promotes hepatocellular carcinoma progression. J Cell Biochem 118: 4836-4843, 2017.

14. Zhang HM, Yang FQ, Chen SJ, Che J and Zheng JH: Upregulation of long non-coding RNA MALAT1 correlates with tumor progression and poor prognosis in clear cell renal cell carcinoma. Tumour Biol 36: 2947-2955, 2015.

15. Xiong Z, Wang L, Wang $Q$ and Yuan Y: LncRNA MALAT1/miR-129 axis promotes glioma tumorigenesis by targeting SOX2. J Cell Mol Med, May 29, 2018 (Epub ahead of print).

16. Latouche C, Natoli A, Reddy-Luthmoodoo M, Heywood SE, Armitage JA and Kingwell BA: MicroRNA-194 modulates glucose metabolism and its skeletal muscle expression is reduced in diabetes. PLoS One 11: e0155108, 2016.

17. Jia Y, Guan M, Zheng Z, Zhang Q, Tang C, Xu W, Xiao Z, Wang $L$ and Xue Y: miRNAs in urine extracellular vesicles as predictors of early-stage diabetic nephropathy. J Diabetes Res 2016: 7932765, 2016.

18. Kong L, Sun M, Jiang Z, Li L and Lu B: MicroRNA-194 inhibits lipopolysaccharide-induced inflammatory response in nucleus pulposus cells of the intervertebral disc by targeting TNF receptor-associated factor 6 (TRAF6). Med Sci Monit 24: 3056-3067, 2018.

19. Wang H, Yu Y, Fan S and Luo L: Knockdown of long non-coding RNA NEAT1 inhibits proliferation and invasion and induces apoptosis of osteosarcoma by inhibiting miR-194 expression. Yonsei Med J 58: 1092-1100, 2017

20. Zhang X, Wei C, Li J, Liu J and Qu J: MicroRNA-194 represses glioma cell epithelialtomesenchymal transition by targeting Bmi1. Oncol Rep 37: 1593-1600, 2017. 
21. Wang ZH, Ren LL, Zheng P, Zheng HM, Yu YN, Wang JL, Lin YW, Chen YX, Ge ZZ, Chen XY, et al: miR-194 as a predictor for adenoma recurrence in patients with advanced colorectal adenoma after polypectomy. Cancer Prev Res (Phila) 7: 607-616, 2014.

22. Mi J, Zou Y, Lin X, Lu J, Liu X, Zhao H, Ye X, Hu H, Jiang B, Han B, et al: Dysregulation of the miR-194-CUL4B negative feedback loop drives tumorigenesis in non-small-cell lung carcinoma. Mol Oncol 11: 305-319, 2017.

23. Zhang M, Zhuang Q and Cui L: MiR-194 inhibits cell proliferation and invasion via repression of RAP2B in bladder cancer. Biomed Pharmacother 80: 268-275, 2016.

24. Li JH, Liu S, Zhou H, Qu LH and Yang JH: starBase v2.0: Decoding miRNA-ceRNA, miRNA-ncRNA and protein-RNA interaction networks from large-scale CLIP-Seq data. Nucleic Acids Res 42 (Database Issue): D92-D97, 2014.

25. Agarwal V, Bell GW, Nam JW and Bartel DP: Predicting effective microRNA target sites in mammalian mRNAs. Elife 4, 2015

26. Zhang M, Liu J, Li M, Zhang S, Lu Y, Liang Y, Zhao K and Li Y: Insulin-like growth factor $1 /$ insulin-like growth factor 1 receptor signaling protects against cell apoptosis through the PI3K/AKT pathway in glioblastoma cells. Exp Ther Med 16: 1477-1482, 2018.

27. Guan J, Zhou Y, Mao F, Lin Y, Shen S, Zhang Y and Sun Q: MicroRNA-320a suppresses tumor cell growth and invasion of human breast cancer by targeting insulin-like growth factor 1 receptor. Oncol Rep 40: 849-858, 2018.

28. Moroishi T, Hansen CG and Guan KL: The emerging roles of YAP and TAZ in cancer. Nat Rev Cancer 15: 73-79, 2015.

29. Song S, Honjo S, Jin J, Chang SS, Scott AW, Chen Q, Kalhor N, Correa AM, Hofstetter WL, Albarracin CT, et al: The hippo coactivator YAP1 mediates EGFR overexpression and confers chemoresistance in esophageal cancer. Clin Cancer Res 21: 2580-2590, 2015.

30. Gu Y, Cai R, Zhang C, Xue Y, Pan Y, Wang J and Zhang Z: miR-132-3p boosts caveolae-mediated transcellular transport in glioma endothelial cells by targeting PTEN/PI3K/PKB/Src/Cav-1 signaling pathway. FASEB J 33: 441-454, 2019.

31. Livak KJ and Schmittgen TD: Analysis of relative gene expression data using real-time quantitative PCR and the 2(-Delta Delta C(T)) method. Methods 25: 402-408, 2001.

32. Xue W, Chen J, Liu X, Gong W, Zheng J, Guo X, Liu Y, Liu L, Ma J, Wang P, et al: PVT1 regulates the malignant behaviors of human glioma cells by targeting miR-190a-5p and miR-488-3p Biochim Biophys Acta Mol Basis Dis 1864: 1783-1794, 2018

33. Wang J, Sun J and Yang F: The role of long non-coding RNA H19 in breast cancer. Oncol Lett 19: 7-16, 2020.

34. Zhang R, Xia Y, Wang Z, Zheng J, Chen Y, Li X, Wang Y and Ming H: Serum long non coding RNA MALAT-1 protected by exosomes is up-regulated and promotes cell proliferation and migration in non-small cell lung cancer. Biochem Biophys Res Commun 490: 406-414, 2017.

35. Lee NK, Lee JH, Ivan C, Ling H, Zhang X, Park CH, Calin GA and Lee SK: MALAT1 promoted invasiveness of gastric adenocarcinoma. BMC Cancer 17: 46, 2017.

36. Pa M, Naizaer G, Seyiti A and Kuerbang G: Long noncoding RNA MALAT1 functions as a sponge of MiR-200c in ovarian cancer. Oncol Res, Sep 11, 2017 (Epub ahead of print).

37. Chang SM and Hu WW: Long non-coding RNA MALAT1 promotes oral squamous cell carcinoma development via microRNA-125b/STAT3 axis. J Cell Physiol 233: 3384-3396, 2018.

38. Handa H, Kuroda Y, Kimura K, Masuda Y, Hattori H, Alkebsi L, Matsumoto M, Kasamatsu T, Kobayashi N, Tahara KI, et al: Long non-coding RNA MALAT1 is an inducible stress response gene associated with extramedullary spread and poor prognosis of multiple myeloma. Br J Haematol 179: 449-460, 2017.

39. Fang Z, Zhang S, Wang Y, Shen S, Wang F, Hao Y, Li Y, Zhang B, Zhou Y and Yang H: Long non-coding RNA MALAT-1 modulates metastatic potential of tongue squamous cell carcinomas partially through the regulation of small proline rich proteins. BMC Cancer 16: 706, 2016.

40. Li L, Chen H, Gao Y, Wang YW, Zhang GQ, Pan SH, Ji L, Kong R, Wang G, Jia YH, et al: Long noncoding RNA MALAT1 promotes aggressive pancreatic cancer proliferation and metastasis via the stimulation of autophagy. Mol Cancer Ther 15 2232-2243, 2016.

41. Huang C, Yu Z, Yang H and Lin Y: Increased MALAT1 expression predicts poor prognosis in esophageal cancer patients. Biomed Pharmacother 83: 8-13, 2016.
42. Miao Y, Fan R, Chen L and Qian H: Clinical significance of long non-coding RNA MALAT1 expression in tissue and serum of breast cancer. Ann Clin Lab Sci 46: 418-424, 2016.

43. Zheng HT, Shi DB, Wang YW, Li XX, Xu Y, Tripathi P, Gu WL, Cai GX and Cai SJ: High expression of lncRNA MALAT1 suggests a biomarker of poor prognosis in colorectal cancer. Int J Clin Exp Pathol 7: 3174-3181, 2014.

44. Li H, Yuan X, Yan D, Li D, Guan F, Dong Y, Wang H, Liu X and Yang B: Long non-coding RNA MALAT1 decreases the sensitivity of resistant glioblastoma cell lines to temozolomide. Cell Physiol Biochem 42: 1192-1201, 2017.

45. Zhao X, Hou Y, Tuo Z and Wei F: Application values of miR-194 and miR-29 in the diagnosis and prognosis of gastric cancer. Exp Ther Med 15: 4179-4184, 2018.

46. Cai Y, Yan P, Zhang G, Yang W, Wang H and Cheng X: Long non-coding RNA TP73-AS1 sponges miR-194 to promote colorectal cancer cell proliferation, migration and invasion via up-regulating TGF $\alpha$. Cancer Biomark 23: 145-156, 2018.

47. Qu F and Cao P: Long noncoding RNA SOX2OT contributes to gastric cancer progression by sponging miR-194-5p from AKT2 Exp Cell Res 369: 187-196, 2018.

48. Chen Y, Wei H, Liu Y and Zheng S: Promotional effect of microRNA-194 on breast cancer cells via targeting F-box/WD repeat-containing protein 7. Oncol Lett 15: 4439-4444, 2018.

49. Jiang Y, Chen J and Chen G: Long noncoding RNA IRAIN acts as tumor suppressor via miR-125b in multiple myeloma. Oncol Lett 18: 6787-6794, 2019.

50. Sun B, Liu C, Li H, Zhang L, Luo G, Liang S and Lü M: Research progress on the interactions between long non-coding RNAs and microRNAs in human cancer. Oncol Lett 19: 595-605, 2020.

51. Yan W, Wu Q, Yao W, Li Y, Liu Y, Yuan J, Han R, Yang J, Ji X and Ni C: MiR-503 modulates epithelial-mesenchymal transition in silica-induced pulmonary fibrosis by targeting PI3K p85 and is sponged by lncRNA MALAT1. Sci Rep 7: 11313, 2017.

52. Stavast CJ and Erkeland SJ: The non-canonical aspects of MicroRNAs: Many roads to gene regulation. Cells 8: pii: E1465, 2019.

53. Zhang B, Li Y, Hou D, Shi Q, Yang S and Li Q: MicroRNA-375 inhibits growth and enhances radiosensitivity in oral squamous cell carcinoma by targeting insulin like growth factor 1 receptor. Cell Physiol Biochem 42: 2105-2117, 2017.

54. Dale OT, Aleksic T, Shah KA, Han C, Mehanna H, Rapozo DC, Sheard JD, Goodyear P, Upile NS, Robinson M, et al: IGF-1R expression is associated with HPV-negative status and adverse survival in head and neck squamous cell cancer. Carcinogenesis 36: 648-655, 2015.

55. Kato H, Sekine Y, Furuya Y, Miyazawa Y, Koike H and Suzuki K: Metformin inhibits the proliferation of human prostate cancer PC-3 cells via the downregulation of insulin-like growth factor 1 receptor. Biochem Biophys Res Commun 461: 115-121, 2015.

56. Deng WY,Li N, Wan XB,Luo SX and Zhang YW: Phosphorylated insulin-like growth factor-1 receptor expression predicts poor prognosis of Chinese patients with gastric cancer. Med Oncol 31: $141,2014$.

57. Wu J, Zhang S, Shan J, Hu Z, Liu X, Chen L, Ren X, Yao L, Sheng $\mathrm{H}, \mathrm{Li} \mathrm{L}$, et al: Elevated HMGA2 expression is associated with cancer aggressiveness and predicts poor outcome in breast cancer. Cancer Lett 376: 284-292, 2016.

58. Luo J, Wu J, Li Z, Qin H, Wang B, Wong TS, Yang W, Fu QL and Lei W: miR-375 suppresses IGF1R expression and contributes to inhibition of cell progression in laryngeal squamous cell carcinoma. Biomed Res Int 2014: 374598, 2014.

59. Zhao $\mathrm{H}$ and $\mathrm{Gu} \mathrm{X}$ : Silencing of insulin-like growth factor-1 receptor enhances the radiation sensitivity of human esophageal squamous cell carcinoma in vitro and in vivo. World J Surg Oncol 12: 325, 2014

60. Eun YG, Lee D, Lee YC, Sohn BH, Kim EH, Yim SY, Kwon KH and Lee JS: Clinical significance of YAP1 activation in head and neck squamous cell carcinoma. Oncotarget 8: 111130-111143, 2017.

61. Jerhammar F, Johansson AC, Ceder R, Welander J, Jansson A, Grafström RC, Söderkvist P and Roberg K: YAP1 is a potential biomarker for cetuximab resistance in head and neck cancer. Oral Oncol 50: 832-839, 2014.

This work is licensed under a Creative Commons Attribution-NonCommercial-NoDerivatives 4.0 International (CC BY-NC-ND 4.0) License. 